the conference. Most of the workers concerned with the practical problems of eliminating odours prefer to use subjective methods; but there is here also a full discussion of objective methods. It has been suggested that the olfactory mucosa is sensitive to concentrations as low as $10^{-13}$; if this is true, there is some considerable way to go, and much fundamental research is required, before adequate objective methods are available. But this is not to deprecate the very valuable and important work of the presentday pioneers.

H. SosNowICK

\section{AGE OF PARENT AND CHARACTER OF OFFSPRING}

$\mathrm{T}$ HE relations of an offspring to its parents are complex, and the ways in which a parent may influence the character of its offspring are many. This is especially true among the higher animals; but even when we turn to lower animals and to plants the parent-offspring relation, if simpler, is still far from simple. Though, as genetics has emphasized, the primary endowment is the complement of nuclear genes, an individual also receives from its parents its initial cytoplasm-mainly, but not necessarily exclusively, from its mother. The cytoplasm so received may contain particles of a genetic or semi-genetic nature (plasmagenes of various kinds), nutritional materials, protective substances and agents of disease (such as viruses). It is a complex endowment as yet by no means fully understood. Nor is the cytoplasm the only avenue of supply of food materials, protective substances and disease risks. The close association of offspring with parent during early life, especially in higher animals, plays its part in the nutrition, protection and disease relation of the offspring and, of course, also in its education.

Our understanding of these various links between the generations is not equal. Classically the geneticist has concerned himself primarily with the nucleus and the genes it carries, setting other aspects aside as secondary to the main problem. Initially this was an essential restriction, but of recent years especially the study of the nuclear genes has itself guided attention to the cytoplasm, to the immunological relations between mother and child, and to the effects of maternal nutrition; indeed, to the whole complexity of the parent-offspring relation. Thus as the genetical framework has become clear, the way has opened up to a co-operative attack on the broader problem by all the many interests which are concerned.

Both the complexity of the problem and the many types of approach which can be made to it are brought out by a recent symposium on "Parental Age and Characteristics of the Offspring" held by the New York Academy of Sciences*. Although in his introduction E. V. Cowdry chiefly emphasizes the effects of age of mother and father on longevity in the offspring, he recognizes in his summary that this provides only a superficial common denominator for the many phenomena described in the nineteen contributions to the symposium, and he stresses the need for a better understanding of the mechanisms which underlie the effects we observe. The observations reported cover a very wide field. Asexually produced progeny in plants are discussed alongside partheno* "Parental Age and Characteristics of the Offspring", edit. L. C.
Strong. Ann. N. Y. Acad. Sci., 57, 451-614 (1954). genetic rotifers and the sexual offspring of mammals. Longevity, germination characters of seeds, mutationrates, the expression of congenital malformation in men and mice, mongolism and the sex ratio in man, fibrocarcinoma and leukæmia in mice, the consequences of ageing gametes before fertilization, skeletal characters, spotting, the results of altering a mother's physiology during pregnancy, and seasonal influences, are all to be found among the subjects considered. As might be expected, the importance of the mother's age emerges clearly as opposed to that of the father; but there is, again not surprisingly, little pattern to be discerned as yet even at the level of description of the phenomena. Suggestions can be, and are freely, made as to the underlying causation. Hormonal changes, cytoplasmic entities, antibody levels, viral effects, biochemical adjustments, and alterations in crossingover with age, are all brought in, and the susceptibility of polygenically determined variants to non-nuclear effects is emphasized. But the value of this symposium lies in the problems it poses and the clues it offers rather than in the explanations it achieves or the mechanisms it exposes.

Two papers are especially interesting in their bearing on the nature of somatic ageing. The first, by A. I. Lansing, describes experiments with the rotifer Philodrina citrina, which, being parthenogenetic, is unlikely to show any significant amount of variation mediated by nuclear genes within its progenies. Separate lines were developed, differing in the age of the mother at the time of production of the eggs from which the next generation was raised. With eggs from adolescent mothers the longevity of the offspring tended to increase as the generations went by. The lines in which eggs were taken from middle-aged or senile mothers, on the other hand, showed a decline in longevity until they died out after five and three generations, respectively. The change is reversible by reversal of the selection. Furthermore, there is a close relation between growth and ageing, the ageing factor appearing in the eggs at the time when the mother ceases growth. Accelerated ageing accelerates growth but decreases maximal size, while retarded ageing retards growth and increases maximal size.

In the second paper E. Ashby and E. Wangerman describe experiments with clones of the water-plant Lemna minor. The fronds of this plant reproduce by asexual buds, the daughters so produced being successively smaller as the mother frond ages. It appears from extrapolation that the age of mother at which daughter size would be zero is the age of death. Thus the meristems grow old with the mother. As with the rotifers the process is cumulative and reversible, for first daughters are larger than their mothers where these mothers were themselves from old parents and therefore small.

The regularity and similarity of behaviour in the rotifer and the plant are striking. Though clearly not dependent on genetic segregation, the responses to selection resemble in several respects those which can be obtained with polygenic variation. Can they depend on a multi-particulate cytoplasmic system, related ultimately to the nucleus, but capable of change independently of the nuclear genes ? Though it is too early to do more than speculate, these papers suggest an experimental approach which might materially advance our understanding of the changes which go on during development and ageing.

K. MATHER 